\title{
Antibiotic sensitivity and RAPD-PCR studies on cultivable gut bacteria from Indian Medicinal Leech-Hirudinaria granulosa
}

\author{
Dhaval Patel ${ }^{*}$, Khushbu Koriya, Pinkal Patel, Jyoti Solanki, Suresh Mesara and M. Nataraj
}

\begin{abstract}
Background: Hirudo granulosa - an Indian cattle leech, is frequently used for the treatment of psoriasis and eczema. During treatment which followed by leech biting, it transfers some amount of gut microbes along with saliva. This may result in bacterial infection at the treatment site. Antibiotics used as post-surgical hirudotherapy is the reason for drug resistance. Drug resistance is the result of the change in the genetic makeup of bacteria. Therefore, it is necessary to study antibiotic sensitivity of gut bacteria and characterized them genetically.

Results: Fourteen bacterial isolates were obtained from unused leech in which five were Gram-negative and the other nine were Gram-positive. Similarly from the used leech, thirteen were isolated in which five were Gramnegative and the other eight were Gram-positive. Biochemical analysis reveals that isolates from unused leech saliva belong to Pseudomonas, Micrococcus, Streptococcus, and Vibrio species, while the used leech salivary bacteria were the member of genus Pseudomonas, Comamonas, Escherichia, Citrobacter, Aeromonas, Providencia, Enterobacter, and Yersinia. Antibiotic sensitivity tests for isolates indicated that chloramphenicol $(30 \mu \mathrm{g})$ and norfloxacin $(10 \mu \mathrm{g})$ were effective for unused leech isolates while sparfloxacin $(5 \mu \mathrm{g})$ and Cefaclor $(30 \mu \mathrm{g})$ were effective against used leech salivary isolates. Random primer (OPL-14)-based random amplified polymorphic DNA (RAPD) fingerprint showed twenty amplified regions among all tested bacteria. Most of the bacteria contain the tested sequence except U2, T2, T9A, and T10 which showed no amplification indicated the absence of primers sequence.
\end{abstract}

Conclusion: Chloramphenicol, norfloxacin, sparfloxacin, and Cefaclor antibiotics alone or in combination were possibly used to treat post-therapy infections. Bacteria from treated and untreated leeches were clustered at nearby branch in neighbor end-joining phylogenetic tree, which indicates the similar (but not exact) genetic makeup. Therefore, it can be concluded that these antibiotics were possibly used against most of them.

Keywords: Antibiotics, Bacterial Infection, Indian Medicinal Leech, Multidrug resistance Phylogenetic tree, Random amplified polymorphic DNA

\section{Background}

The use of living organisms for skin treatment is one of the many techniques since a long time. It includes the skin wound healing practice using leech (Pereira \& Bártolo, 2016). Food and Drug Administration (FDA) approved the use of leech for clinical purposes such as compromised vasculature, since 2004 (Whitaker, Maltz, Siddall, \& Graf, 2014). Pain reduction in osteoarthritis and other joint ailments are more common in leech

\footnotetext{
* Correspondence: dhaval30103@gmail.com

Department of Biosciences, satellite campus, Bakrol-Vadtal Road, Sardar Patel University, Vallabh Vidyanagar, Anand 388 315, India
}

therapy. Other medicinal treatment includes cardiovascular diseases, hemorrhoids, and reconstructive surgeries (Das, 2014). Leech is the segmented annelids and known for its sanguivorous nature (Das, 2014). It belongs to phylum Annelids; class Clitellata, subclass Hirudinea (Lamarck, 1818). In general, treatment includes leech biting followed by blood-sucking activity of leech which is painless due to salivary bioactive compound hirustasin (Das, 2014; Sig, Guney, Uskudar Guclu, \& Ozmen, 2017). The saliva of medicinal leech contains many bioactive compounds include anticoagulant, regulating factors for thrombin and platelet inhibition, which prevent 
the blood from coagulation during feeding (Sig et al., 2017).

Digestion of blood is completed through the action of many digestive enzymes and complex microbial community present in the gut of animal which influences the metabolism of an animal. These organisms absorb nutrients from the lumen and survive in the digestive tract condition. Moreover, they create a barrier for other microbes and prevent their proliferation on the surface (Graf, 2016). Leech biting transfers saliva along with some amount of gut microbes (Giltner, Bobenchik, Uslan, et al., 2013). Complications like bacterial infections in leech therapy were reported in $2 \%$ to $36 \%$ cases (Marden, McClure, Beka, \& Graf, 2016). Such infectious bacteria considered to have come from the gut of the leech. Gut microbes reside in the mucosal membrane of the digestive tract of the leech and they transmit through saliva (Whitaker et al., 2014). Gut microbial community explains immunity of animal (here leech) and also provide information against the pathogen colonization. Moreover, gut microbes play an important role in symbiosis with host animal and also help in the digestion of nutrients (Worthen, Gode, \& Graf, 2006).

Antimicrobial resistance is a serious problem nowadays for the humans, which occured due to the extensive use of antimicrobial agents for human welfare (Beka, Fullmer, Colston, et al., 2018). Medicinal leech is treated with a prophylactic antibiotic before application for medicinal purposes. These antibiotics reduced the chances of several bacterial infections (Marden et al., 2016). It triggers the chance of multidrug resistance (MDR) and also the transmission of such MDR bacteria among the hosts (Giltner et al., 2013). Therefore, antibiotics assay and genetic characterization of gut microbes become important to conserve such multitasking therapeutic invertebrates. In the present study, an attempt was made to isolate cultivable bacteria from leech saliva and their antibiotic assay in order to propose the broad-spectrum antibiotic for promising prophylaxis. Molecular characterization by RAPD-PCR was also carried out to evaluate random sequence-based evolutionary relationship analysis.

\section{Materials and methods}

\section{Organism of the study}

Indian medicinal leech Hirudinaria granulosa were collected from Ahmadabad (Surekh Education, M85/ 1009, Panchvati apartment, Sola Road, Naranpura, opp. Rameshwar app., Near Indian oil petrol pump, between Bhuyandev to Parasnagar, Ahmadabad). Leech was divided into two categories. One, before any skin treatment named as 'unused' (Coded as UTL or $\mathrm{U})$ while the second was after skin disease treatment named as 'used' (coded as TL or T) for the present study.

\section{Culture maintenance}

Leeches were stored and maintained in a transparent glass container. The container should be sufficiently big enough and filled up pure dechlorinated tap water up to 3/4th of its capacity, allowing enough space for the movement of leech. The water was changed after two successive days. The lid of the container must contain fine holes for air exchange.

\section{Preparation of leech for experiment}

The purification of leeches was carried out by putting the leeches in dechlorinated water containing a pinch of turmeric for $60 \mathrm{~min}$ to increase the appetite and bloodsucking power.

\section{Saliva extraction from used and unused leach}

Both used and unused leeches were starved for 3 weeks before the extraction of saliva. Starved leeches were fed with phagostimulatory solution [0.01 M Arginine in 0.15 $\mathrm{M}$ sodium chloride kept at $37^{\circ} \mathrm{C}$ ] through sterile glass funnel wrapped with ultra-violet (UV) sterilized parafilm sheets (Bemis, Neenah WI 54956). Leeches were introduced near the parafilm sheet and allowed attaching itself. After the leech becomes fully satiated, it detached itself from the parafilm. Leeches were kept in sterile ice container for $15-20 \mathrm{~min}$, during the incubation time, leech became completely paralyzed and regurgitated all the solution it had already sucked. Then leeches were squeezed from the posterior end toward the anterior end in order to collect the remaining sucked solution under sterile condition. Collected saliva was centrifuged at $10,000 \mathrm{rpm}$ for $15 \mathrm{~min}$. Sediment was used for bacterial isolation. Leeches regained their activity on immersing them in warm dechlorinated water $\left(37^{\circ} \mathrm{C}\right)$ for $15-30 \mathrm{~min}$.

\section{Isolation of gut/saliva microflora}

The bacterial suspension was serially diluted in sterile distilled water up to $10^{(-10)}$ dilution and $0.1 \mathrm{ml}$ of inoculum was spread on the nutrient agar plate. Inoculated media plates were incubated at $37^{\circ} \mathrm{C}$ for $24 \mathrm{~h}$. Colonies with different morphological appearances were picked up after incubation.

\section{Gram staining and biochemical test for bacterial isolates}

All bacterial isolates collected from the saliva of unused and used leech were categorized by Gram staining (Hucker's modification). All the Gram-positive bacteria were inoculated with commercially available biochemical identification API kit (HiMedia product code: KB013). 
Table 1 Colonial character of bacterial isolates from unused leech saliva

\begin{tabular}{|c|c|c|c|c|c|c|c|}
\hline Characters & Size & Shape & Edge & Elevation & Surface & Opacity & Pigment \\
\hline UTL 01 & Intermediate & Round & Entire & Flat & Smooth \& punctate & Oleaginous & Orangish yellow \\
\hline UTL 02 & Large & Irregular & Lobate & Effused & Smooth \& echinate & Cretaceous & No pigment \\
\hline UTL 03 & Intermediate & Mycelioid & Filamentous & Flat & Smooth \& squamous & Oleaginous & Creamish yellow \\
\hline UTL 04 & Intermediate & Elliptical & Undulated & Effused & Smooth \& squamous & Oleaginous & Blue-green \\
\hline UTL 05 & Large & Elliptical & Articulate & Flat & Smooth \& squamous & Resinous & Brown \\
\hline UTL 06 & Intermediate & Elliptical & Fimbriate & Effused & Smooth \& papilate & Transparent & No pigment \\
\hline UTL 07 & Large & Round & Articulate & Umbonate & Smooth \& papilate & Vitreous & No pigment \\
\hline UTL 08 & Large & Round & Articulate & Convex & Smooth \& bullet & Oleaginous & Brick red \\
\hline UTL 09 & Intermediate & Round & Articulate & Effused & Smooth \& squamous & Oleaginous & Orange \\
\hline UTL 10 & Intermediate & Elliptical & Repand & Flat & Smooth \& squamous & Resinous & Brown \\
\hline UTL 11 & Intermediate & Round & Entire & Effused & Smooth \& punctate & Cretaceous & No pigment \\
\hline UTL 12 & Large & Round & Entire & Flat & Smooth \& punctate & Oleaginous & No pigment \\
\hline UTL 13 & Small & Punctiform & Entire & Umbonate & Smooth \& papilate & Cretaceous & No pigment \\
\hline UTL 14 & Intermediate & Round & Entire & Flat & Smooth \& punctate & Transparent & No pigment \\
\hline
\end{tabular}

Similarly, Gram-negative bacteria were inoculated on API kit (HiMedia product code: KB002) for rapid identification of the organism. Both kits were incubated at $37^{\circ} \mathrm{C}$ for $48 \mathrm{~h}$.

\section{Antibiotic assays}

Antibiotic assays of bacterial isolates were carried out using universal antibiotic combo disc (IC001* procured from HiMedia, Mumbai). For assays, each bacterial culture was grown in nutrient broth until $\mathrm{OD}_{600}$ reaches up to 1.00. Next day, bacterial culture was spread on nutrient agar medium prepared in $20-\mathrm{cm}$ Petri plate and the antibiotic disc was placed on the surface of the medium after inoculation. Plates were incubated at $37^{\circ} \mathrm{C}$ overnight and next day, plates were checked for zone of inhibition.
[*Antibiotics on disc: norfloxacin $(10 \mu \mathrm{g})$, gentamicin $(10 \mu \mathrm{g})$, chloramphenicol $(30 \mu \mathrm{g})$, cefuroxime $(30 \mu \mathrm{g})$, ciprofloxacin $(5 \mu \mathrm{g})$, cefoperazone $(75 \mu \mathrm{g})$, ceftazidime $(30 \mu \mathrm{g})$, roxithromycin $(30 \mu \mathrm{g})$, clarithromycin $(15 \mu \mathrm{g})$, cotrimoxazole $(25 \mu \mathrm{g})$, netillin $(30 \mu \mathrm{g})$, cefaclor $(30 \mu \mathrm{g})$, cefadroxil $(30 \mu \mathrm{g})$, azithromycin $(15 \mu \mathrm{g})$, Amphicilli/Cloxacillin $(10 \mu \mathrm{g})$, Penicillin-G (10 units), amikacin $(30 \mu \mathrm{g})$, sparfloxacin $(5 \mu \mathrm{g})$, Ampicillin/Sulbactam $(10 / 10 \mu \mathrm{g})]$

\section{DNA isolation and quantification}

Bacterial genomic DNA was extracted by the modified Green and Sambrook method for bacterial genomic DNA extraction (Green \& Sambrook, 2012). Briefly, bacterial (both Gram-negative and Gram-positive) cells were harvested by centrifugation at $8000 \mathrm{rpm}$ for $10 \mathrm{~min}$. Pellet obtained was re-suspended in $300 \mu$ of SET buffer

Table 2 Colonial morphology of bacterial isolates from used leech saliva

\begin{tabular}{|c|c|c|c|c|c|c|c|}
\hline Characters & Size & Shape & Edge & Elevation & Surface & Opacity & Pigment \\
\hline TL 01 & Intermediate & Round & Erose & Effused & Smooth \& punctate & Oleaginous & Blue-green \\
\hline TL 02 & Large & Fusiform & Auriculate & Flat & Smooth \& punctate & Oleaginous & Blue-green \\
\hline TL 03 & Intermediate & Punctiform & Entire & Effused & Smooth \& Punctate & Cretaceous & No pigment \\
\hline TL 04 & Intermediate & Elliptical & Lobate & Effused & Smooth \& squamous & Oleaginous & Blue-green \\
\hline TL 05 & Intermediate & Round & Entire & Umbonate & Smooth \& papillate & Cretaceous & No pigment \\
\hline TL 06 & Large & Elliptical & Erose & Umbilicate & Smooth \& punctate & Resinous & Brown \\
\hline TL 07 & Large & Fusiform & Auriculate & Raised & Smooth \& alveolate & Oleaginous & Blue-green \\
\hline TL 08 & Intermediate & Round & Repand & Raised & Smooth \& squamous & Resinous & Brown \\
\hline TL 09 & Large & Round & Auriculate & Convex & Smooth \& papillate & Cretaceous & Orange-red \\
\hline TL 10 & Intermediate & Irregular & Auriculate & Raised & Smooth \& punctate & Cretaceous & No pigment \\
\hline TL 11 & Small & Punctiform & Entire & Umbonate & Smooth \& papillate & Cretaceous & No pigment \\
\hline TL 12 & Large & Irregular & Auriculate & Umbonate & Smooth \& contoured & Sebaceous & No pigment \\
\hline TL 13 & Intermediate & Elliptical & Fimbriate & Raised & Smooth \& Echinate & Resinous & Brownish white \\
\hline
\end{tabular}


Table 3 Morphological characterization of isolates from unused leech under 100x magnification

\begin{tabular}{lllll}
\hline Sr. no. & Code & Gram reaction & Morphological characters (100x) & Motility \\
\hline 1 & UTL01 & Gram-negative & Bacilli in clusters & Non-motile \\
2 & UTL02 & Gram-positive & Big rod in short chain & Motile \\
3 & UTL03 & Gram-positive & Bacilli in chains & Motile \\
4 & UTL04 & Gram-positive & Rode shape in cluster & Motile \\
5 & UTL05 & Gram-negative & Rode shape in cluster & Brownian \\
6 & UTL06 & Gram-positive & Cocci in clusters & Motile \\
7 & UTL07 & Gram-positive & Rod shape in clusters & Motile \\
8 & UTL08 & Gram-positive & Rod scattered & Motile \\
9 & UTL09 & Gram-negative & Rods in clusters & Non-motile \\
10 & UTL10 & Gram-negative & Cocci, scattered & Non-motile \\
11 & UTL11 & Gram-positive & Big rod, single \& scattered & Motile \\
12 & UTL12 & Gram-positive & Rods in clusters & Motile \\
14 & UTL13 & Gram-negative & Bacilli in clusters & \\
\hline
\end{tabular}

(made up of $2 \mathrm{ml} 1 \mathrm{M}$ Tris, $1 \mathrm{ml} 5 \mathrm{M} \mathrm{NaCl}$, and $0.2 \mathrm{ml}$ $0.5 \mathrm{M}$ ethylenediaminetetraacetic acid (EDTA) in the final volume of $100 \mathrm{ml}$ in double-distilled water) followed by incubation at $37^{\circ} \mathrm{C}$ for an hour. In order to break open the cells and remove RNA from mixture, $10 \mu \mathrm{l}$ of lysozyme and RNase each were added to it respectively. Mixture was incubated at $37^{\circ} \mathrm{C}$ for $90 \mathrm{~min}$ and later, $23 \mu \mathrm{l}$ sodium dodecyl sulfate (SDS) and $10 \mu \mathrm{l}$ Proteinase- $\mathrm{K}$ was added and kept at $55^{\circ} \mathrm{C}$ for 2 -h incubation. Later, $85 \mu$ of $5 \mathrm{M} \mathrm{NaCl}$ was added and after 5 min $85 \mu \mathrm{l}$ Chloroform was added followed by mixing by inverting the tunes. Mixture was kept at room temperature for $45 \mathrm{~min}$ and then centrifuged at 10,000 rpm for $10 \mathrm{~min}$ to separate phases. Aqueous phaseupper phase was transferred to a fresh 1.5-ml Eppendorf tube and added equal volume of $80 \%$ ethanol to precipitate DNA. Allow tubes to air dry under laminar airflow. DNA was resuspended in $40 \mu$ tris-EDTA (TE) Buffer. DNA concentrations obtained from each bacterial sample were quantified (in $\mathrm{ng} / \mu \mathrm{l}$ ) spectrophotometrically using UV-visible spectrophotometer (Shimadzu UV VIS 1800 -A114548) by checking absorption at $260 \mathrm{~nm}$ and $280 \mathrm{~nm}$ followed by calculating ratio $\left(\mathrm{OD}_{260} / \mathrm{OD}_{280}\right)$.

\section{RAPD-PCR}

The purity of extracted DNA was checked by separating on $1 \%$ agarose gel. One $\mu \mathrm{l}$ of (15 ng purified) genomic DNA and $2.7 \mu$ l Master Mix (EmeraldAmp GT PCR Master mix-2X Premix, Takara, Tokyo) were taken in

Table 4 Morphological characterization of isolates form used leech under 100x magnification

\begin{tabular}{lllll}
\hline Sr. no. & Code & Gram reaction & Characters & Motility \\
\hline 1 & TL01 & Gram-positive & Rod in cluster & Motile \\
2 & TL02 & Gram-negative & Bacilli in Single \& Cluster & Motile \\
3 & TL03 & Gram-negative & Bacilli in Cluster \& Duplicate & Motile \\
4 & TL04 & Gram-Positive & Rod in clusters and short chains & Motile \\
5 & TL05 & Gram-negative & Bacilli, scattered & Motile \\
6 & TL06 & Gram-positive & Rode, scattered & Motile \\
7 & TL07 & Gram-positive & Rod, single & Motile \\
8 & TL08 & Gram-negative & Bacilli, scattered & Non-motile \\
10 & TL09 & Gram-positive & Cocci in clusters & Motile \\
11 & TL10 & Gram-positive & Bacilli in clusters & Motile \\
12 & TL11 & Gram-POSITIVE & Big rod in chains & Motile \\
\hline
\end{tabular}


Table 5 Biochemical characterizations of unused leech Gram-positive isolates

\begin{tabular}{|c|c|c|c|c|c|c|c|c|c|c|}
\hline Sr.no. & $\begin{array}{l}\text { Isolates } \rightarrow \\
\text { Name of test } \\
\downarrow\end{array}$ & UTL02 & UTL03 & UTL04 & UTL06 & UTL07 & UTL08 & UTL11 & UTL12 & UTL14 \\
\hline 1 & $\begin{array}{l}\text { Malanate } \\
\text { production }\end{array}$ & $-V e$ & $+v e$ & + ve & $-V e$ & $-\mathrm{Ve}$ & $-V e$ & $-V e$ & $-V e$ & $+v e$ \\
\hline 2 & V.P. test & -Ve & -Ve & $-V e$ & $-V e$ & -Ve & $-V e$ & $-V e$ & $+v e$ & -Ve \\
\hline 3 & $\begin{array}{l}\text { Citrate } \\
\text { utilization }\end{array}$ & -Ve & + ve & +ve & +ve & +ve & $-V e$ & $-V e$ & +ve & $+v e$ \\
\hline 4 & ONPG test & -Ve & $-V e$ & $-V e$ & -Ve & $-V e$ & $-V e$ & $-V e$ & $-V e$ & -Ve \\
\hline 5 & $\begin{array}{l}\text { Nitrate } \\
\text { reduction }\end{array}$ & $-V e$ & -Ve & $+V e$ & $-V e$ & $-V e$ & $-V e$ & $-V e$ & $+v e$ & $+v e$ \\
\hline 6 & Catalase test & $-V e$ & + ve & +ve & $-V e$ & + ve & $-V e$ & $-V e$ & + ve & + ve \\
\hline 7 & Arginine test & $-V e$ & +ve & +ve & $+v e$ & +ve & $-V e$ & $-V e$ & $-V e$ & $+v e$ \\
\hline 8 & $\begin{array}{l}\text { Sucrose } \\
\text { utilization }\end{array}$ & -Ve & $-V e$ & $-V e$ & +ve & $-V e$ & $-V e$ & $-V e$ & $+v e$ & -Ve \\
\hline 9 & $\begin{array}{l}\text { Mannitol } \\
\text { utilization }\end{array}$ & -Ve & $-V e$ & $-V e$ & $-V e$ & -Ve & $-V e$ & $-V e$ & $-V e$ & $-V e$ \\
\hline 10 & $\begin{array}{l}\text { Glucose } \\
\text { utilization }\end{array}$ & +ve & + ve & $-V e$ & $+v e$ & -Ve & $-V e$ & $-V e$ & $+v e$ & $+v e$ \\
\hline 11 & $\begin{array}{l}\text { Arabenose } \\
\text { utilization }\end{array}$ & $-V e$ & $+v e$ & $+v e$ & $-V e$ & $-V e$ & + ve & $-V e$ & $-V e$ & + ve \\
\hline 12 & $\begin{array}{l}\text { Trehalose } \\
\text { utilization }\end{array}$ & +ve & $-V e$ & $-V e$ & $+v e$ & $-V e$ & $-V e$ & $-V e$ & + ve & $-V e$ \\
\hline 13 & MR test & $-V_{e}$ & + ve & $-V e$ & $-V_{e}$ & + ve & $+v e$ & -Ve & $-V e$ & +ve \\
\hline 14 & $\begin{array}{l}\text { Oxidase } \\
\text { production }\end{array}$ & +ve & +ve & +ve & + ve & $-V e$ & -Ve & +ve & $+v e$ & +ve \\
\hline 15 & $\mathrm{H}_{2} \mathrm{~S}$ test & $-V e$ & $-V_{e}$ & $-V e$ & $-V e$ & $-V e$ & + ve & $-V_{e}$ & $-V e$ & $-V e$ \\
\hline 16 & $\begin{array}{l}\text { Gelatin } \\
\text { hydrolysis }\end{array}$ & $-V e$ & + ve & $-V e$ & $-V e$ & +ve & + ve & + ve & + ve & + ve \\
\hline 17 & $\begin{array}{l}\text { Starch } \\
\text { hydrolysis }\end{array}$ & +ve & + ve & $+V e$ & $+v e$ & +ve & + ve & + ve & $-V e$ & + ve \\
\hline 18 & $\begin{array}{l}\text { Casein } \\
\text { hydrolysis }\end{array}$ & $-V e$ & $-V_{e}$ & +ve & $-V e$ & +ve & + ve & $-V e$ & $-V e$ & $-V e$ \\
\hline \multicolumn{2}{|c|}{ Probable bacteria } & $\begin{array}{l}\text { Micrococcus } \\
\text { lylae }\end{array}$ & $\begin{array}{l}\text { Micrococcus } \\
\text { luteus }\end{array}$ & $\begin{array}{l}\text { Bacillus } \\
\text { cereus }\end{array}$ & $\begin{array}{l}\text { Streptococcus } \\
\text { group F }\end{array}$ & $\begin{array}{l}\text { Bacillus } \\
\text { megaterium }\end{array}$ & $\begin{array}{l}\text { Bacillus } \\
\text { coagulans }\end{array}$ & $\begin{array}{l}\text { Micrococcus } \\
\text { lylae }\end{array}$ & $\begin{array}{l}\text { Bacillus } \\
\text { cereus }\end{array}$ & $\begin{array}{l}\text { Bacillus } \\
\text { megaterium }\end{array}$ \\
\hline
\end{tabular}

fresh PCR tube along with $0.6 \mu \mathrm{l}$ of OPL-14 (random) primer [5'-TCG CTC CGT T-3'] and $5.7 \mu \mathrm{l}$ sterile distilled water was added to get the final volume of reaction mixture to $10 \mu \mathrm{l}$. PCR amplification was done by keeping $94{ }^{\circ} \mathrm{C}$ for $1 \mathrm{~min}$ as initial denaturation step followed by $37^{\circ} \mathrm{C}$ annealing for $1 \mathrm{~min}, 40$ cycles of extension at $72^{\circ} \mathrm{C}$ with 2 min duration and final extension for $10 \mathrm{~min}$ again at $72{ }^{\circ} \mathrm{C}$ (Padmalatha \& Prasad, 2006).

\section{Agarose gel electrophoresis}

Agarose gel (1\%) prepared in TAE buffer (1X) was used to check the purity and RAPD pattern analysis. Two $\mu$ lof PCR amplicon was used for electrophoresis with $1 \mathrm{~kb}$ ladder agarose gel containing EtBr $(5 \mu \mathrm{l} / \mathrm{ml}$ of gel $)$ at $60 \mathrm{~V}$. DNA banding pattern was visualized in UV trans-illuminator and was documented using BioRed Gel documentation system,
Molecular Imager ${ }^{\oplus}$ ChemiDoc ${ }^{\mathrm{Tm}} \mathrm{XRS}^{+}$equipped with Image $\mathrm{Lab}^{\mathrm{Tm}}$ Software version 6.0.0 Bio-Red Laboratories Inc.

\section{Phylogenetic tree construction by neighbor end-joining method}

Phylogenetic relationships between isolates were evaluated by constructing the phylogenetic tree using PyElph GUI software version 1.4, according to the neighbor end-joining method.

\section{Results}

Isolation and morphological characterization of isolates Fourteen bacterial isolates with different morphological characters were obtained from the saliva of unused leech which was coded as UTL-1 to UTL-14. Colonial characters observed visually are described in Table 1 . From used leech, thirteen bacterial isolates obtained were 
Table 6 Biochemical characterization of Gram-negative isolates from unused leech

\begin{tabular}{|c|c|c|c|c|c|c|}
\hline \multirow{2}{*}{$\begin{array}{l}\text { Sr. } \\
\text { no }\end{array}$} & Isolates $\rightarrow$ & UTL01 & UTL05 & UTL09 & UTL10 & UTL14 \\
\hline & \multicolumn{6}{|l|}{ Name of test $\downarrow$} \\
\hline 1 & Citrate utilization & -ve & +ve & $-v e$ & +ve & -ve \\
\hline 2 & Lysine test & -ve & -ve & $-v e$ & $-v e$ & -ve \\
\hline 3 & Ornithin test & -ve & -ve & -ve & -ve & -ve \\
\hline 4 & Urease production & +ve & -ve & +ve & +ve & +ve \\
\hline 5 & Phenylalanine & +ve & $-v e$ & $-v e$ & $-v e$ & +ve \\
\hline 6 & Nitrate reduction & +ve & $-v e$ & +ve & $-v e$ & +ve \\
\hline 7 & $\mathrm{H}_{2} \mathrm{~S}$ production & -ve & -ve & +ve & -ve & -ve \\
\hline 8 & Glucose utilization & +ve & $-v e$ & $-v e$ & +ve & +ve \\
\hline 9 & Adonitol utilization & -ve & $-v e$ & $-v e$ & $-v e$ & $-v e$ \\
\hline 10 & Lactose utilization & $-v e$ & $-v e$ & $-v e$ & $-v e$ & -ve \\
\hline 11 & Arabinose utilization & -ve & $-v e$ & +ve & +ve & $-v e$ \\
\hline 12 & Sorbitol utilization & -ve & $-v e$ & -ve & $-v e$ & -ve \\
\hline 13 & MR test & +ve & -ve & $-v e$ & +ve & $-v e$ \\
\hline 14 & VP test & $-v e$ & $-V e$ & $-v e$ & +ve & -ve \\
\hline 15 & Oxidase test & +ve & +ve & +ve & +ve & +ve \\
\hline 16 & Gelatin hydrolysis & -ve & $-V e$ & +ve & +ve & +ve \\
\hline 17 & Starch hydrolysis & -ve & $-V e$ & -ve & +ve & -ve \\
\hline 18 & Casein hydrolysis & -ve & $-V e$ & -ve & +ve & +ve \\
\hline \multicolumn{2}{|c|}{ Probable bacteria } & P. stutzeri or P. mesophilica & Alcaligenes faecalis & P. putrefaciens & Aeromonas hydrophila & Vibrio. Marinus \\
\hline
\end{tabular}

Table 7 Biochemical characterization of Gram-negative isolates from used leech

\begin{tabular}{|c|c|c|c|c|c|c|}
\hline \multirow{2}{*}{$\begin{array}{l}\text { Sr. } \\
\text { no. }\end{array}$} & Isolates $\rightarrow$ & TL02 & TL03 & TL05 & TL08 & TL13 \\
\hline & \multicolumn{6}{|l|}{ Name of test $\downarrow$} \\
\hline 1 & Citrate utilization & $+v e$ & -ve & $+v e$ & +ve & $+v e$ \\
\hline 2 & Lysine utilization & -ve & -ve & -ve & -ve & -ve \\
\hline 3 & Ornithin test & -ve & -ve & -ve & $-v e$ & +ve \\
\hline 4 & Urease production & -ve & -ve & $+v e$ & +ve & +ve \\
\hline 5 & Phenylalanine utilization & -ve & -ve & -ve & +ve & + ve \\
\hline 6 & Nitrate reduction & -ve & -ve & -ve & -ve & -ve \\
\hline 7 & $\mathrm{H}_{2} \mathrm{~S}$ test & -ve & -ve & -ve & -ve & $+v e$ \\
\hline 8 & Glucose utilization & -ve & -ve & $+v e$ & +ve & +ve \\
\hline 9 & Adonitol utilization & -ve & -ve & -ve & -ve & -ve \\
\hline 10 & Lactose utilization & -ve & -ve & + ve & -ve & -ve \\
\hline 11 & Arabinose utilization & $-v e$ & -ve & +ve & $+v e$ & $+v e$ \\
\hline 12 & Sorbitol utilization & -ve & -ve & -ve & -ve & -ve \\
\hline 13 & MR test & -ve & $+v e$ & $-v e$ & $-v e$ & -ve \\
\hline 14 & VP test & -ve & -ve & -ve & $-v e$ & -ve \\
\hline 15 & Oxidase production & $+v e$ & $+v e$ & -ve & +ve & $+v e$ \\
\hline 16 & Gelatin hydrolysis & -ve & $+\mathrm{ve}$ & $+v e$ & $-v e$ & -ve \\
\hline 17 & Starch hydrolysis & $+v e$ & $+v e$ & $-v e$ & $-v e$ & +ve \\
\hline 18 & Casein hydrolysis & -ve & -ve & -ve & +ve & +ve \\
\hline \multicolumn{2}{|c|}{ Probable bacteria } & P. putida & $\begin{array}{l}\text { V. salmonicida or } \\
\text { E. coli(inactive) }\end{array}$ & $\begin{array}{l}\text { Providencia rettgeri or } \\
\text { Ent. agglomerans }\end{array}$ & Burkhoderia gladioli & Citrobacter diversus \\
\hline
\end{tabular}


Table 8 Biochemical characterization of used leech Gram-positive isolates

\begin{tabular}{|c|c|c|c|c|c|c|c|c|c|}
\hline $\begin{array}{l}\text { Sr. } \\
\text { no. }\end{array}$ & $\begin{array}{l}\text { Isolates } \rightarrow \\
\text { Name of Test } \downarrow\end{array}$ & TL01 & TL04 & TL06 & TL07 & TL09 & TL10 & TL11 & TL12 \\
\hline 1 & $\begin{array}{l}\text { Malonate } \\
\text { utilization }\end{array}$ & +ve & +ve & +ve & +ve & -ve & -ve & -ve & -ve \\
\hline 2 & V.P. test & -ve & -ve & -ve & -ve & -ve & +ve & -ve & +ve \\
\hline 3 & Citrate utilization & +ve & +ve & +ve & +ve & +ve & -ve & +ve & -ve \\
\hline 4 & ONPG test & -ve & -ve & -ve & -ve & -ve & -ve & -ve & -ve \\
\hline 5 & Nitrate reduction & -ve & -ve & -ve & -ve & -ve & -ve & -ve & +ve \\
\hline 6 & $\begin{array}{l}\text { Catalase } \\
\text { production }\end{array}$ & +ve & $+\mathrm{ve}$ & +ve & +ve & +ve & +ve & -ve & +ve \\
\hline 7 & Arginine test & +ve & +ve & +ve & +ve & +ve & -ve & -ve & +ve \\
\hline 8 & $\begin{array}{l}\text { Sucrose } \\
\text { utilization }\end{array}$ & -ve & -ve & -ve & -ve & -ve & +ve & -ve & +ve \\
\hline 9 & $\begin{array}{l}\text { Mannitol } \\
\text { utilization }\end{array}$ & -ve & -ve & -ve & -ve & -ve & +ve & -ve & +ve \\
\hline 10 & $\begin{array}{l}\text { Glucose } \\
\text { utilization }\end{array}$ & -ve & $+v e$ & +ve & +ve & -ve & +ve & +ve & +ve \\
\hline 11 & $\begin{array}{l}\text { Arabenose } \\
\text { utilization }\end{array}$ & +ve & -ve & -ve & -ve & -ve & -ve & +ve & -ve \\
\hline 12 & $\begin{array}{l}\text { Thehalose } \\
\text { utilization }\end{array}$ & -ve & -ve & -ve & -ve & -ve & -ve & -ve & -ve \\
\hline 13 & MR test & -ve & -ve & +ve & -ve & -ve & +ve & -ve & -ve \\
\hline 14 & Oxidase test & +ve & +ve & -ve & +ve & -ve & +ve & +ve & $+v e$ \\
\hline 15 & $\mathrm{H}_{2} \mathrm{~S}$ production & -ve & -ve & -ve & -ve & -ve & -ve & -ve & -ve \\
\hline 16 & Gelatin hydrolysis & -ve & +ve & +ve & +ve & -ve & -ve & +ve & +ve \\
\hline 17 & Starch hydrolysis & +ve & + ve & +ve & +ve & -ve & +ve & -ve & -ve \\
\hline 18 & Casein hydrolysis & -ve & -ve & +ve & +ve & -ve & +ve & -ve & -ve \\
\hline \multicolumn{2}{|c|}{ Probable bacteria } & $\begin{array}{l}\text { Bacillus } \\
\text { megaterium }\end{array}$ & $\begin{array}{l}\text { Bacillus } \\
\text { megaterium }\end{array}$ & $\begin{array}{l}\text { Corynebacterium } \\
\text { spps. }\end{array}$ & $\begin{array}{l}\text { Flavobacterium } \\
\text { spps. }\end{array}$ & $\begin{array}{l}\text { Bacillus } \\
\text { spps. }\end{array}$ & $\begin{array}{l}\text { S. } \\
\text { cohnii }\end{array}$ & $\begin{array}{l}\text { Bacillus } \\
\text { pumilus }\end{array}$ & $\begin{array}{l}\text { Bacillus } \\
\text { coagulans }\end{array}$ \\
\hline
\end{tabular}

coded as TL-1 to TL-13 and colonial characteristic of each isolated were presented in Table 2. Each of the isolates was categorized according to their Gram's reaction.

Out of fourteen isolated from unused leech, five were Gram-negative while the other nine were Gram-positive. Gram-negative used leech salivary isolates were five while rests of eight were Gram-positive (Tables 3 and 4).

\section{Biochemical characterization of isolates}

Bacterial cultures isolated from leech were categorized as Gram-positive and Gram-negative. Biochemical characterization of each isolates were described in Tables 5, 6, 7 and 8. Positive biochemical tests of each isolates were compared with the manual provided with identification kit for preliminary identification. The list of probably identified bacteria was also included in Tables 5, 6, 7 and 8 .

Bacteria isolated from unused leech were quite different from those found in used leech. Interestingly, it was observed that certain species of bacteria which were not present initially become part of the digestive tract of leech after treatment. In the present experiment, leech was starved for 15 days to remove possible bacteria which were carrying temporarily. Bacillus species was found in both types of leech saliva more commonly. Pseudomonas species were present higher in number before the treatment. Micrococcus species which was found in unused leech which completely disappears in used leech. Vibrio species were also found in both the leech.

\section{Antibiotic assay of isolates}

The antibiotic assay was carried out for the bacteria isolated from the leech saliva (Figs. 1 and 2). Clear zone around the antibiotic disc indicated that bacteria were sensitive at a given concentration. It was observed that all isolates were sensitive to most of the antibiotics. In general, chloramphenicol $(30 \mu \mathrm{g})$ and norfloxacin $(10 \mu \mathrm{g})$ showed highest sensitivity for bacteria from unused leech. While bacteria isolated from used leech showed the highest sensitivity towards sparfloxacin $(5 \mu \mathrm{g})$ followed by cefaclor $(30 \mu \mathrm{g})$. 

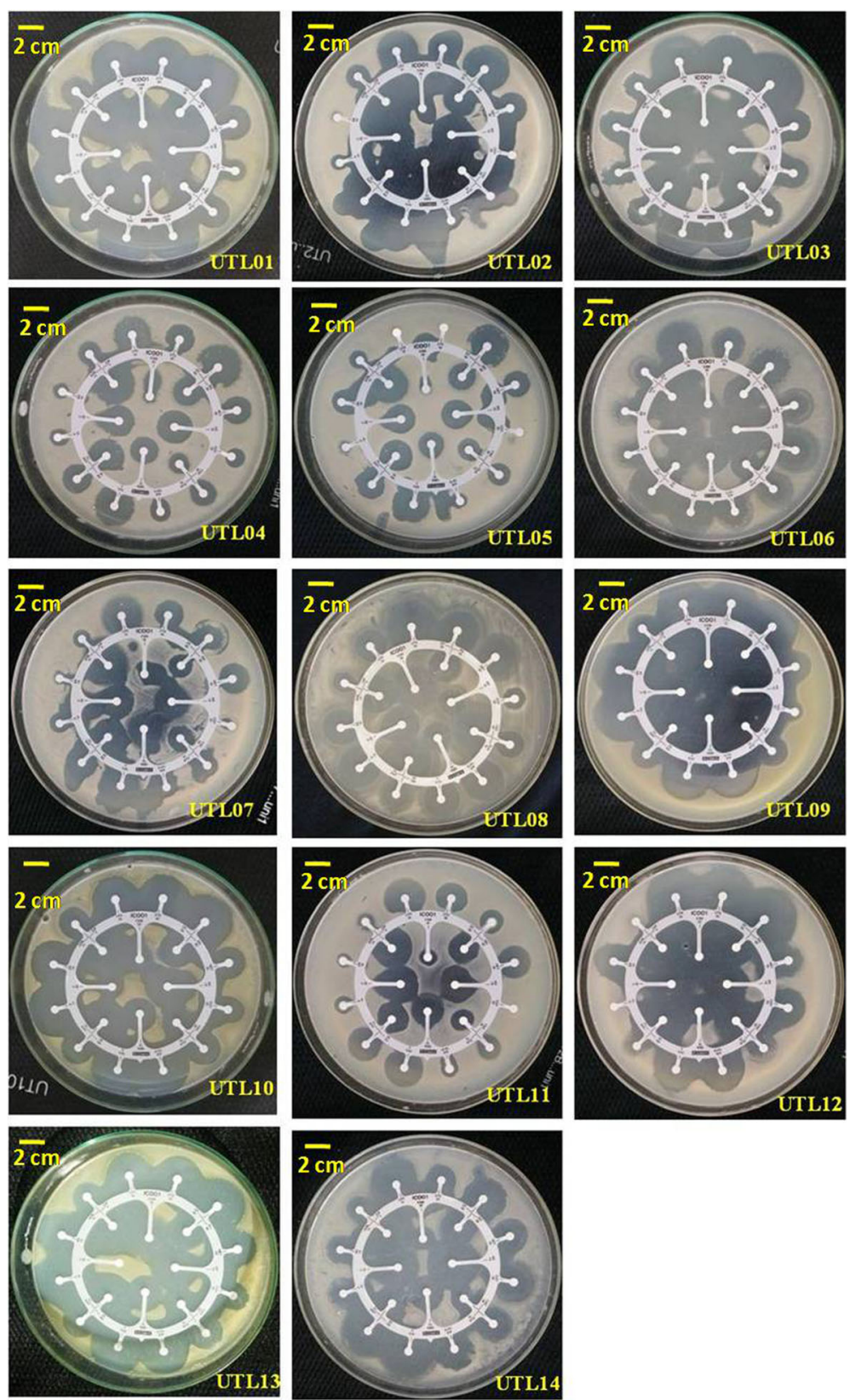

Fig. 1 Antimicrobial assay of bacteria from untreated leech

\section{RAPD-PCR and phylogenetic tree}

In the present study, RAPD-PCR was performed using random primer OPL-14 to screen intra-species variation among the isolates from both used and unused leech. Variation was screened with respect to random primer OPL-14 (Chacon, Martinez, Wachsman, et al., 2013; Fernanda, Da, Biscola, et al., 2016). There were 20 amplified band patterns were observed using OPAL14 primer (Fig 3 ). The variation in band patterns for each successfully characterized isolate as indicated by RAPD-PCR possibly suggests the existence of sequence within the species
(Adeleke, Olaitan, Abiona, et al., 2014). Figure 3 showed the RAPD band pattern with a ladder $(1 \mathrm{~kb})$ at the first lane.

Phylogenetic tree construction (Fig. 4) by neighbor end-joining method reveals that isolate U7, U4, and U3 were phylogenetically different than U11, U8, U10, U9, U12, and U6. Similarly, T9B, T8, T12, and T10 contain different genetic makeup than T4 and T1. Branches of these isolates indicated that they were evolved to survive under similar conditions. U2, T2, T9A, and T10 showed no amplification, which indicated absence of primers sequence. 

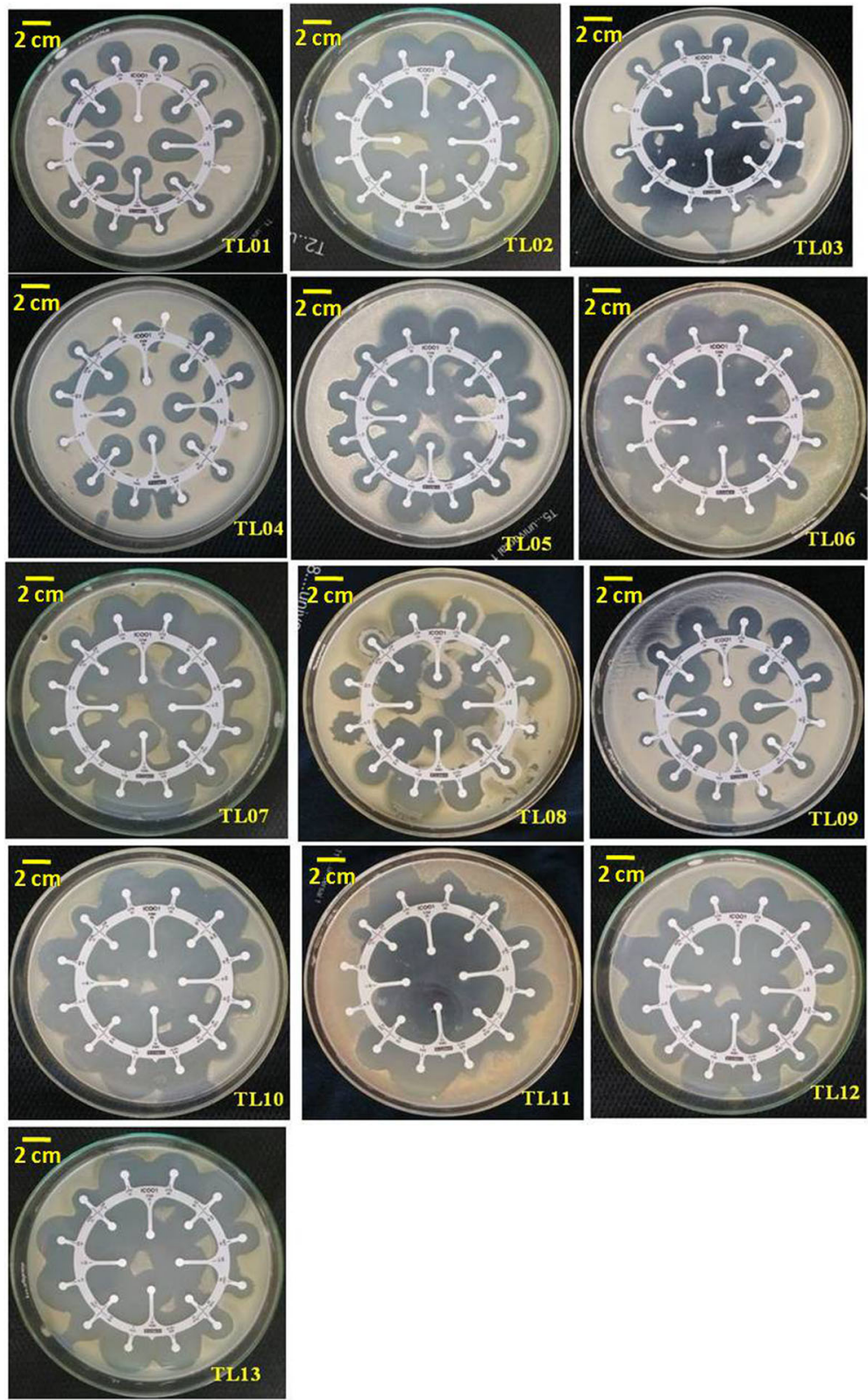

Fig. 2 Antibiotic assay of bacteria isolated from treated leech

\section{Discussion}

Herudotherapy or leech therapy for skin diseases is an ancient process (Das, 2014). These blood-sucking animals serve the human kind for since long. The saliva of medicinal leech contains many bioactive compounds and it was reviewed in detail in the past years (Siddall, Trontelj, Utevsky, Nkamany, \& Macdonald-III, 2007; Das, 2014; Sig et al., 2017). These bioactive compounds are of many medicinal properties for which leeches were exploited (Siddall et al., 2007). But saliva does not only contain bioactive compounds but it is also the habitat of some gut microbes. Such gut microbes are normal flora of them or might becomes from the environment in which the leeches live. These microfloras might be pathogenic to humans. For example, Aeromonas which is commensal in the leech digestive system is pathogenic for human (Marden et al., 2016). It reported to cause pneumonia, gastroenteritis, and septicemia (Das, 2014). Therefore, medicinal leech, before the treatment of skin diseases, treated with antibiotic solutions to eliminate possibilities of post-operational bacterial infections (Das, 2014). Microevolution process changes the genetics of these bacteria as they divide in a very short time and the problem of drug resistance arises. Nowadays, due to the 


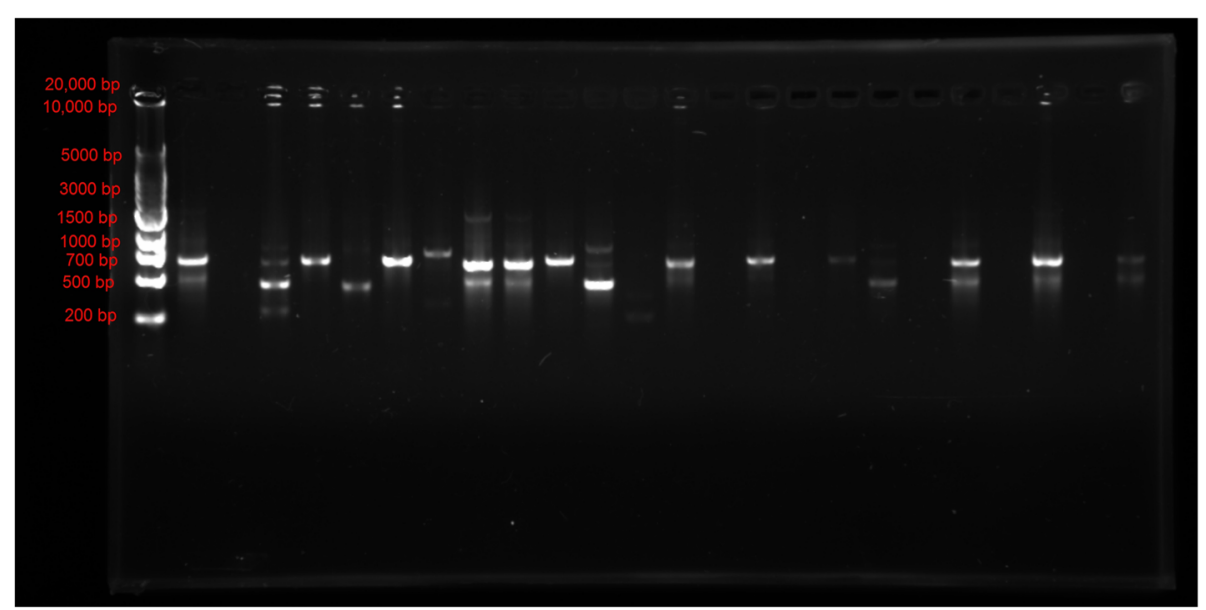

Fig. 3 RAPD fingerprint of all isolates using OPL-14 as a random primer

excessive use of many antibiotics, these drug resistance phenomena changed in multidrug resistance (MDR). The bacterium which was sensitive to a certain drug becomes resistant to those antibiotics. MDR become the global threat (Giltner et al., 2013). MDR is a genetic phenomenon. Therefore, it becomes important to evaluate the salivary microbiota for their genetic profile and antibiotic sensitivity. Conventional microbiological techniques were used to isolate the bacteria from the saliva of the leech. For the present study, two types of leech were used. One leech was before the skin treatment and another was after the skin treatment. Bacterial isolates were also tested biochemically to find out the genus name of them. It was noted that salivary bacterial makeup was partially changed. Aeromonas and Burkholderia were isolated from used leech which was thought to be present in the digestive tract of leech as symbiotic bacteria (Graf, 2016; Ott, Dacks, Ryan, \& Rio, 2016;

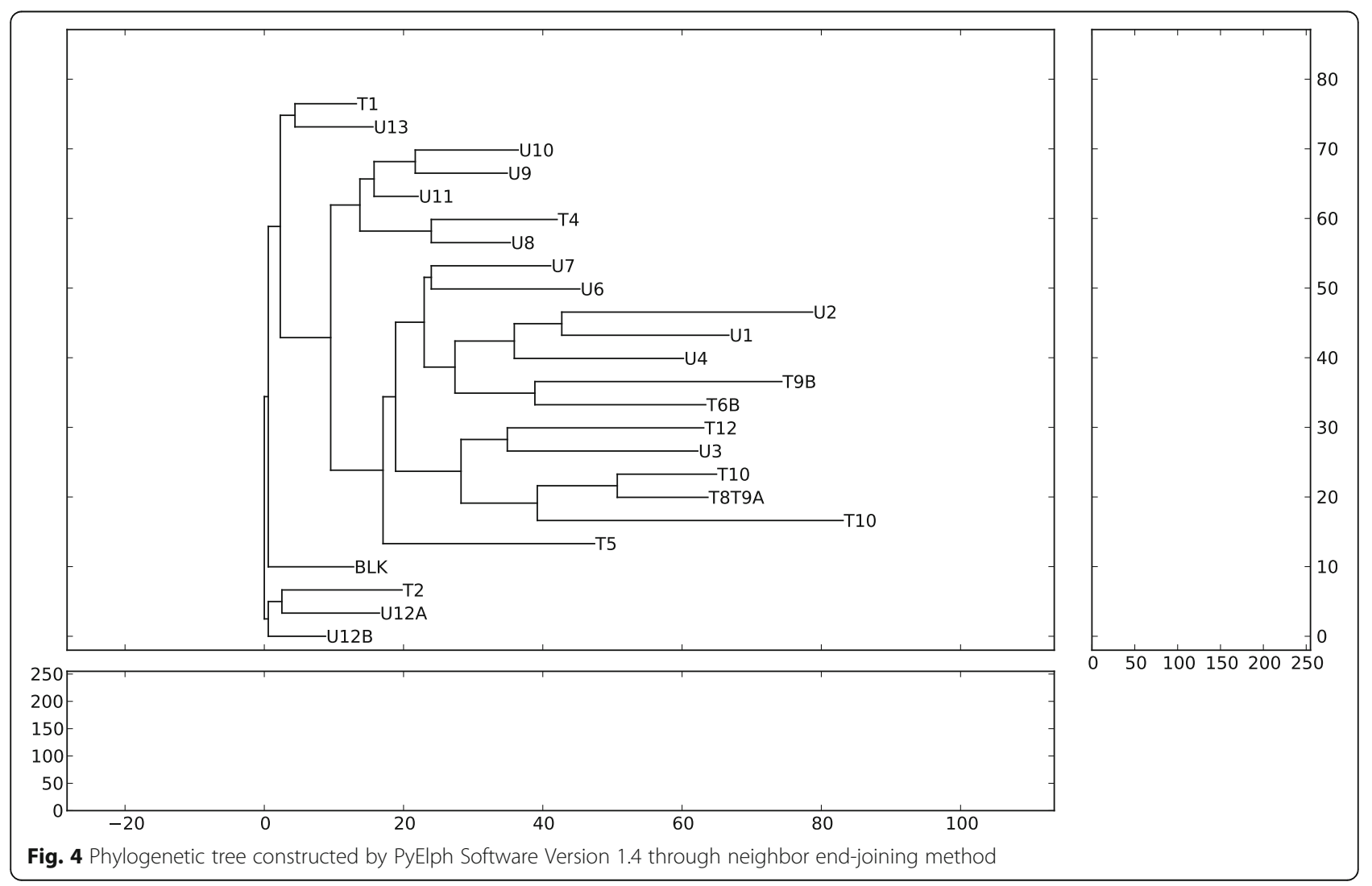


Paoletti, Mazzon, Martinez-Sañudo, et al., 2013; Worthen et al., 2006). Leech received bacteria that belong to genera Citrobacter, Comamonas, Corynebacterium, Providencia, Escherichia, and Enterobacter. Burkholderia present in the midgut of many crypts (Paoletti et al., 2013). Bacteria which belong to Escherichia and Enterobacter might have come from water in which leech was maintained. Comamonas was isolated from the mucosal secretion of $H$. verbena (Ott, Rickards, Gehrke, \& Rio, 2014).

Antibiotic sensitivity of all of them was analyzed by disc diffusion technique using a commercially available antibiotic combo disc containing 20 antibiotics. The technique is equally important because it also gives the comparative results of each antibiotic tested. Each bacterium was sensitive to most of the antibiotics. Most of the bacterial infections which resulted from leech therapies were due to Vibrio, Pseudomonas, and Aeromonas. These infections would be treated with oral antibiotics, for example, fluoroquinolones, trimethoprim, aminoglycosides with cephalosporins-3rd generation (Das, 2014; Gunawan, Wibowo, Bunawan, \& Turner, 2015). Cefepime, cotrimoxazole, vancomycin, co-trimoxazole, aztreonam, ampicillin and metronidazole were reported as treatment for Aeromonas infection developed due to leech therapy (Giltner et al., 2013; Marden et al., 2016). Recently, Aeromonas species resistant from fluoroquinolones and ciprofloxacin was isolated (Beka et al., 2018) which raised the need of a new combination of known antibiotics for the treatment.

The RAPD technique constitutes an efficient tool for the study of DNA polymorphism. It involves the amplification of random segments of genomic DNA by PCR using short-single primers of arbitrary sequence. RAPD requires very small quantities of DNA while no cloning, sequencing or hybridization is necessary. The possible number of RAPD patterns was estimated by the changes of one or more clear bands or band size. The RAPD technique, therefore, surveys (scans) numerous loci in the genome, which makes this method particularly attractive for analyzing the genetic distance and similarity between closely related species (Ling et al. 2007). Phylogenetic tree on the basis of OPL-14 confirmed that the bacterial genera isolated from each leech were able to survive in the same environmental conditions. The change in genetic makeup expressed as a new branch in phylogenetic tree.

\section{Conclusion}

Bacterial culture isolated from unused and used leech were morphologically, biochemically, and genetically characterized. Present study was conducted to isolate the cultivable bacterial culture from 'unused' and 'used' Indian medicinal leech (Hirudinaria granulose) and characterized them morphologically, biochemically and genetically. It was found that bacterial makeup in both the leech was different. Biochemical characterization reveals that isolates were belong to different genera viz Bacillus, Pseudomonas, Micrococcus, Aeromonas, Citrobacter, Vibrio, Providencia, Escherichia, Enterobacter etc. Bacteria from unused leech were more sensitive to chloramphenicol and norfloxacin while bacteria from used leech showed the highest sensitivity towards sparfloxacin and cefaclor which suggested that these antibiotics may serve as prophylactic for post-leech therapy bacterial infections. RAPD analysis using random primer OPL-14 (5'- TCG CTC CGT T-3') illustrated that most of the genera isolated from both types of leeches contain primer sequence. Most of the bacteria from isolated from each leech showed clustering in phylogenetic tree indicated that they were of similar origin and genetic makeup with respect to tested random primer. Therefore, these antibiotics possibly used against them.

\section{Supplementary information}

Supplementary information accompanies this paper at https://doi.org/10. 1186/s41936-020-00143-5.

Additional file 1. Area of Zone of inhibition of Unused leech isolates in $\mathrm{cm}$. Additional file 2. Area of Zone of inhibition of Used leech isolates in $\mathrm{cm}$.

\section{Abbreviations}

$\mu \mathrm{g}$ : MicrogramEDTAEthylenediaminetetraacetic acidFDAFood and Drug AdministrationMMolarMDRMultidrug resistancePCRPolymerase chain reactionRAPDRandom amplified polymorphic DNArpRotation per minuteSDSSodium dodecyl sulfateTE bufferTris-EDTA bufferUVUltraviolet

\section{Acknowledgements}

Authors are thankful to Surekh Education, M85/1009, Panchvati apartment, Sola Road, Naranpura, opp. Rameshwar app., Near Indian oil petrol pump, between Bhuyandev and Parasnagar, Ahmadabad for help by providing both types of animals, i.e., used and unused leech for the present study. Without their kind support, the study cannot be possible.

\section{Authors' contributions}

DP and JS participated in the design and concept of the study, molecular experimentations, data analysis and drafted the manuscript. KK carried out isolation and biochemical characterization of isolates. JS, PP, and SM were involved in molecular experimentation for RAPD profile and antibiotic sensitivity testing; MN was involved in conception, design, and coordination of the study and also in preparation of the final draft of the manuscript. All authors read and approved the final manuscript.

\section{Funding}

Not applicable.

\section{Availability of data and materials}

The dataset(s) supporting the conclusions of this article is (are) included in the article. The conclusions are based on the data generated from the current study. The author can be contacted for any additional supporting data required by the journal.

Ethics approval and consent to participate

Not applicable as the study relates to the use of leech of this species only. The authors declare that no animal was sacrificed for this study. 


\section{Consent for publication}

Not applicable.

\section{Competing interests}

The authors declare that they have no competing interests.

Received: 21 May 2019 Accepted: 11 February 2020

Published online: 11 March 2020

\section{References}

Adeleke, M. A., Olaitan, J. O., Abiona, O., et al. (2014). Molecular characterization and antibiotic susceptibility patterns of bacteria isolated from wara (west African cheese ) sold in Osun state. Nigeria., 15, 23-30.

Beka, L., Fullmer, M.S., Colston S. M., et al. (2018). Low-level antimicrobials in the medicinal leech select for resistant pathogens that spread to patients. American society for microbiology; mBio, 9, 1-17. doi: 10.1128/mBio.01328-18

Chacon, R., Martinez, R., Wachsman, M., et al. (2013). Biochemical, antimicrobial and molecular characterization of a noncytotoxic bacteriocin produced by Lactobacillus plantarum ST71KS. Food Microbiology, 34, 376-381. https://doi. org/10.1016/j.fm.2013.01.011.

Das, B. K. (2014). An overview on hirudotherapy/leech therapy. Indian Research Journal of Pharmacy and Science, 1, 33-45.

Fernanda, F., Da, P., Biscola, V., et al. (2016). LWT - Food science and technology effect of indigenous lactic acid bacteria isolated from goat milk and cheeses on folate and riboflavin content of fermented goat milk. LWT - Food Science and Technology, 71, 55-161. https://doi.org/10.1016/j.lwt.2016.03.033.

Giltner, C. L., Bobenchik, A. M., Uslan, D. Z., et al. (2013). Ciprofloxacin-resistant Aeromonas hydrophila cellulitis following leech therapy. Journal of Clinical Microbiology, 51, 1324-1326. https://doi.org/10.1128/JCM.03217-12.

Graf, J. (2016). Lessons from digestive-tract symbioses between bacteria and invertebrates. Annual Review of Microbiology, 70, 375-393. https://doi.org/10. 1146/annurev-micro-091014-104258.

Green, M.R., Sambrook, J., (2012). Molecular cloning_a laboratory manual, Fourth Edi. Cold Spring Harbor Laboratory Press.

Gunawan, F., Wibowo, Y. R., Bunawan, N. C., \& Turner, J. H. (2015). Controversy: Hirudotherapy (leech therapy) as an alternative treatment for osteoarthritis. Acta Medica Indonesiana, 47, 176-180.

Lamarck, J.B., (1818). Histoire Naturelle des Animaux sans Vertèbres. Vol. 5. Baillière, Paris, 612 pp

Ling, L., Jie, L., Zou, L., Su-ying, B., Li-ming, N. \& Yu-kun, M. (2007) RAPD analysis of genetic diversity of nine strains of Auricularia auricular cultivated in Heilongjiang Province. Journal of Forestry Research, 18(2), 136-138.

Marden, J. N., McClure, E. A., Beka, L., \& Graf, J. (2016). Host matters: medicinal leech digestive-tract symbionts and their pathogenic potential. Frontiers in Microbiology, 7, 1-11. https://doi.org/10.3389/fmicb.2016.01569.

Ott, B. M., Dacks, A. M., Ryan, K. J., \& Rio, R. V. M. (2016). A tale of transmission: Aeromonas veronii activity within leech-exuded mucus. Applied and Environmental Microbiology, 82, 2644-2655. https://doi.org/10.1128/AEM. 00185-16.

Ott, B. M., Rickards, A., Gehrke, L., \& Rio, R. V. M. (2014). Characterization of shed medicinal leech mucus reveals a diverse microbiota. Frontiers in Microbiology, 5, 1-10. https://doi.org/10.3389/fmicb.2014.00757.

Padmalatha, K., \& Prasad, M. N. V. (2006). Optimization of DNA isolation and PCR protocol for RAPD analysis of selected medicinal and aromatic plants of conservation concern from Peninsular India. African Journal of Biotechnology, 5, 230-234. https://doi.org/10.5897/AJB05.188.

Paoletti, M. G., Mazzon, L., Martinez-Sañudo, I., et al. (2013). A unique midgutassociated bacterial community hosted by the cave beetle Cansiliella servadeii (Coleoptera: Leptodirini) reveals parallel phylogenetic divergences from universal gut-specific ancestors. BMC Microbiology, 13. https://doi.org/ 10.1186/1471-2180-13-129.

Pereira, R. F., \& Bártolo, P. J. (2016). Traditional Therapies for Skin Wound Healing. Advances in Wound Care, 5, 208-229. https://doi.org/10.1089/wound.2013. 0506.

Siddall, M. E., Trontelj, P., Utevsky, S. Y., Nkamany, M., \& Macdonald-III, K. S. (2007). Diver molecular data demonstrate that commercially available medicinal leech are not Hirudo medicinalis. Proceedings of the Royal Society B, 274, 1481-1487. https://doi.org/10.1098/rspb.2007.0248.

Sig, A. K., Guney, M., Uskudar Guclu, A., \& Ozmen, E. (2017). Medicinal leech therapy —an overall perspective. Integrative Medicine Research, 6, 337-343. https://doi.org/10.1016/j.imr.2017.08.001.
Whitaker, I. S., Maltz, M., Siddall, M. E., \& Graf, J. (2014). Characterization of the digestive tract microbiota of Hirudo orientalis (Medicinal Leech) and antibiotic resistance profile. Plastic and Reconstructive Surgery, 133, 408-418. https://doi. org/10.1097/01.prs.0000438461.06217.bb.

Worthen, P. L., Gode, C. J., \& Graf, J. (2006). Culture-independent characterization of the digestive-tract microbiota of the medicinal leech reveals a tripartite symbiosis. Applied and Environmental Microbiology, 72, 4775-4781. https://doi. org/10.1128/AEM.00356-06.

\section{Publisher's Note}

Springer Nature remains neutral with regard to jurisdictional claims in published maps and institutional affiliations.

\section{Submit your manuscript to a SpringerOpen ${ }^{\circ}$ journal and benefit from:}

- Convenient online submission

- Rigorous peer review

- Open access: articles freely available online

- High visibility within the field

- Retaining the copyright to your article

Submit your next manuscript at $\boldsymbol{\nabla}$ springeropen.com 